Canad. Math. Bull. Vol. 19 (3), 1976

\title{
1-COHOMOLOGY AND SPLITTING OF GROUP EXTENSIONS
}

\author{
BY \\ G. N. PANDYA AND R. D. BERCOV
}

The object of this note is to give simpler proofs of a splitting theorem of Gaschütz [1] and a related theorem for groups with operators by using cross-homomorphisms (1-cocycles) instead of 2-cohomology.

We recall that a cross-homomorphism or 1-cocycle from a group $E$ to an abelian normal subgroup $N$ of $E$ is a map $f$ from $E$ to $N$ such that $f(a b)=(f(a))^{b} f(b)$ for all $a, b \in E$ where superscript denotes conjugation. Cocycles $f$ and $h$ are equivalent if for some $n \in N$ we have $h(e)=n^{e} f(e) n^{-1}$ for all $e \in E$.

The set $C^{1}(E, N)$ of all cocycles is an abelian group with respect to pointwise multiplication and the equivalence classes give us the quotient group $H^{1}(E, N)$.

It is not difficult to check that if $f$ is a cocycle which inverts $N$, then $\{e f(e) \mid e \in E\}$ is a complement for $N$ in $E$. Conversely, if $C$ is a complement for $N$ in $E$, the map $f$ defined by $f(c n)=n^{-1}$ is a cocycle. Moreover two complements of $N$ are conjugate by an element of $N$ if and only if the corresponding cocycles are equivalent in the above sense.

The usual proofs of the splitting theorems we discuss here depend on the corestriction map in dimension two. We use the corestriction map in dimension one. Since the existence and elementary properties in dimension one are easily checked, we state them without proof as a lemma:

LEMMA. Let $N$ be an abelian normal subgroup of a group $E$ and $F$ a subgroup of finite index $k$ in $E$ which contains $N$. Then for $f \in C^{1}(F, N)$

(i) there exists $f^{*} \in C^{1}(E, N)$ defined by

$$
f^{*}(e)=\prod_{r \in R} f\left(\mathrm{res}^{-1}\right)^{s}
$$

where $R$ is a right transversal for $F$ in $E$ which contains the group identity and for $r$ in $R, s$ is the element of $R$ for which res $^{-1}$ belongs to $F$.

(ii) if $f(n)=n^{-1}$ then $f^{*}(n)=n^{-k}$ for $n \in N$.

(iii) iff and $h$ are equivalent in $C^{1}(F, N)$, then $f^{*}$ and $h^{*}$ are equivalent in $C^{1}(E, N)$.

(iv) if $f$ is the restriction to $F$ of $h \in C^{1}(E, N)$, then $f^{*}$ and $h^{k}$ are equivalent in $C^{1}(E, N)$.

THEOREM 1. Let $E_{i}, i=1, \ldots, m$ be subgroups of finite index $k_{i}$ in $E$ where for each $i, E_{i}$ contains the abelian normal subgroup $N$ of $E$. If $N$ has a complement in 
each $E_{i}$ and the map $n \rightarrow n^{k}$ has an inverse $\alpha$ on $N$, where $k$ is the greatest common divisor of the $k_{i}$, then

(i) $N$ has a complement in $E$.

(ii) if for each $i$ all complements of $N$ in $E_{i}$ are conjugate in $E_{i}$, then all complements of $N$ in $E$ are conjugate in $E$.

Proof. (i) Because $N$ has a complement in $E_{i}$ we have an $f_{i} \in C^{1}\left(E_{i}, N\right)$ which inverts $N$. If we choose integers $m_{i}$ with $k=\sum_{i=1}^{m} k_{i} m_{i}$ it is easy to see that the map $h$ from $E$ to $N$ defined by $h(e)=\prod_{i}\left(f_{i}^{*}(e)\right)^{m_{i}}$ belongs to $C^{1}(E, N)$ and maps $n \in N$ to $n^{-k}$. Since $\alpha$, being the inverse of the $k$ th power map, commutes with conjugation, the composite $\alpha \circ h$ is also in $C^{1}(E, N)$ and inverts $N$; so $N$ has a complement in $E$.

(ii) It is sufficient to show that any two maps $f, h$ in $C^{1}(E, N)$ are equivalent. By assumption, their restrictions $f_{i}$ and $h_{i}$ to $E_{i}$ are equivalent. Thus by (iii) of the lemma we have that $f_{i}^{*}$ and $h_{i}^{*}$ are equivalent and therefore by (iv) that $f^{k_{i}}$ and $h^{k_{i}}$ are also equivalent. It follows easily that $f^{k}=\prod_{i}\left(f^{k_{i}}\right)^{m_{i}}$ and $h^{k}=\prod_{i}\left(h^{k_{i}}\right)^{m_{i}}$ are equivalent. This means that for some $n \in N$ we have $(f(e))^{k}=n^{e} h(e)^{k} n^{-1}$ for all $e \in E$. It follows that $f(e)=(\alpha(n))^{e} h(e) \alpha(n)^{-1}$, and thus that $f$ and $h$ are equivalent.

COROLlary 1 (Gaschütz [1]). Let $F$ be a subgroup of finite index $k$ in $E$ such that $F$ contains an abelian normal subgroup $N$ of $E$. If $N$ has a complement in $F$ and the map $n \rightarrow n^{k}$ has an inverse $\alpha$ on $N$, then

(i) $N$ has a complement in $E$.

(ii) if all complements of $N$ in $F$ are conjugate in $F$, then all complements of $N$ in $E$ are conjugate.

We now show that one-cocycle proofs also apply for groups with operators.

THEOREM 2. Let A be a group such that

$$
1 \rightarrow N \rightarrow E \rightarrow G \rightarrow 1
$$

is an exact sequence of A-groups where $N$ is abelian. Then (*) splits as an A-sequence if one of the following holds:

(i) $G$ is finite of order $k$, the map $n \rightarrow n^{\kappa}$ has an inverse $\alpha$ on $N$, and $H^{1}(A, N)=1$.

(ii) $G$ is finite of order $k$, the map $n \rightarrow n^{k}$ has an inverse $\alpha$ on $N$, and $A$ acts fixed point freely on $G$.

(iii) $A$ is finite of order $l$, the map $n \rightarrow n^{l}$ has an inverse $\beta$ on $N$, and (*) splits as a sequence of abstract groups.

Proof. (i) Let $\bar{E}$ be the semi-direct product of $A$ and $E$. It is easy to see that $A N$ is of index $k$ in $E$, and thus by the lemma, the $f \in C^{1}(A N, N)$ which corresponds to the complement $A$ of $N$ induces an $f^{*} \in C^{1}(E, N)$ such that $f^{*}(n)=n^{-k}$ for $n \in N$. The composite $h=\alpha \circ f^{*}$ belongs to $C^{1}(\bar{E}, N)$ and inverts $N$. On $A$ we have $h(a)=n^{a} n^{-1}$ for some fixed $n \in N$ since $H^{1}(A, N)=1$. Thus we have $a h(a)=\operatorname{nan}^{-1}$ 
and $a \in C^{n}$ where $C=\{e h(e) \mid e \in \bar{E}\}$ is the complement of $N$ associated with $h$. Since $A$ is contained in $C^{n}$, it follows easily that $E \cap C^{n}$ is an $A$-invariant complement for $N$ in $E$.

(ii) Since $A$ acts fixed point freely on the finite group $E / N$ there will be one orbit, $N$, of length one and some number, $m$, or orbits of length $l$. This means that with $x_{0}=1$ and $x_{i}$ any element from a coset in the $i$ th non-trivial orbit, $\bigcup_{i=0}^{m}\left\{x_{i}^{a} \mid a \in A\right\}$ will be a transversal for $N$ in $E$, hence also for $N A$ in $\bar{E}=E A$. We use this transversal to induce $f^{*} \in C^{1}(\bar{E}, N)$ from the $f \in C^{1}(N A, N)$ associated with the complement $A$. Now for $a \in A$, we have $f^{*}(a)=\prod_{r} f\left(\operatorname{ras}^{-1}\right)^{s}$ where $s$ is the representative of the coset of $r a$. Thus we have $\operatorname{ras}^{-1}=a r^{a} s^{-1}$ in $N A$, and therefore $r^{a} s^{-1}$ in $N A$ also. Since our transversal was chosen to be closed under the action of $A$, we must have $r^{a}=s$ and $\operatorname{ras}^{-1}=a$. Since $f$ was the cocycle associated with the complement $A$, we have $f(a)=1$ and $f^{*}(a)=\prod_{r} 1^{s}=1$. If follows that each $a=a\left(\alpha \circ f^{*}\right)(a)$ belongs to the complement, $C$, associated with $\alpha \circ f^{*}$. As before we have that $C \cap E$ is an $A$-invariant complement for $N$ in $E$.

(iii) By hypothesis we have $f \in C^{1}(E, N)$ which induces $f^{*} \in C^{1}(\bar{E}, N)$ such that $f^{*}(n)=n^{-l}$. Thus $h=\beta \circ f^{*}$ belongs to $C^{1}(\bar{E}, N)$ and inverts $N$. Since $A$ is finite we have from the lemma that $h^{l}$ is equivalent on $A$ to the member of $C^{1}(A, N)$ induced by the restriction of $h$ to the trivial subgroup which is of course trivial. Thus we have for $a \in A$ that $h^{l}(a)=n^{a} n^{-1}$ and $h(a)=(\beta(n))^{a}(\beta(n))^{-1}$ for some $n \in N$. Thus we may adjust $h$ by this coboundary to obtain a cocycle which is trivial on $A$ and whose complement $C$ therefore contains $A$. As before we have that $C \cap E$ is an $A$-invariant complement for $N$ in $E$.

COROLlaRY 2 (Hofmann [2]). Let $N$ be an abelian normal subgroup of a finite group $E$, and suppose that $N$ has a complement in $E$. Let $A$ be a group of automorphisms of $E$ such that $N^{\alpha}=N$, for all $\alpha \in A$, and g.c.d. $(|N|,|A|)=1$. Then $N$ has a complement $C$ in $E$ such that $C^{\alpha}=C$ for all $\alpha \in A$.

COROLlaRY 3 (Maschke, Schur [3, p. 122]). Let $G$ be a finite group of order $l$ and $M a$ G-module with a G-endomorphism $\beta$ such that $\beta(l x)=x$ for all $x \in M$. Let $M=N \oplus K$ be an abelian group direct decomposition of $M$ such that $N$ is a $G$-submodule of $M$. Then there is a $G$-submodule $L$ of $M$ with $M=N \oplus L$.

\section{REFERENCES}

1. W. Gaschütz, Zur Erweiterungstheorie endlicher Gruppen. J. Math. 190, 93-107 (1952).

2. K. H. Hofmann, Zerfallung topologischer Gruppen. Math. Z. 84, 16-37 (1964)

3. B. Hubbert, Endliche Gruppen I, Springer-Verlag (1967).

\section{Department of Mathematics, UNIVERSTTY OF AlBerTa, EDMonton, Alberta, Canada, T6G 2G1.}

